\title{
Comparative Study of Triangulation based and Feature based Image Morphing
}

\author{
Ms. Bhumika G. Bhatt ${ }^{1}$ \\ Assistant Professor Computer Science Engg. Department, VBTIT\&RC, Bardoli ${ }^{1}$ \\ 2009 bhatt@gmail. $\mathrm{com}^{1}$
}

\begin{abstract}
Image Morphing is one of the most powerful Digital Image processing technique, which is used to enhance many multimedia projects, presentations, education and computer based training. It is also used in medical imaging field to recover features not visible in images by establishing correspondence of features among successive pair of scanned images. This paper discuss what morphing is and implementation of Triangulation based morphing Technique and Feature based Image Morphing. IT analyze both morphing techniques in terms of different attributes such as computational complexity, Visual quality of morph obtained and complexity involved in selection of features.
\end{abstract}

\section{KEYWORDS}

Morphing,, Triangulation based Morphing, Field Morphing, Feature based Morphing, Interpolation.

\section{INTRODUCTION}

Morphing is derived from term Metamorphosis means changes in object form [7]. It is a digital image Processing Technique used as animation tool for metamorphosis from one image to another image [3]. Basically, morphing is achieved by coupling image warping with colour interpolation [6].The idea is to specify a warp that distorts the first image into the second. As the morphing proceeds, the source image is gradually distorted and is faded out, while the destination image starts out totally distorted toward the source image and is faded in. early images in sequence more like source image[1][2].

The middle image of the sequence is the average of the source image distorted halfway toward the destination image and the destination image distorted halfway back toward the source image. The last images in the sequence are similar to the destination image. The middle image is key frame, if it looks good then probably the entire morphing sequence will look good [3]. Feature specification is the most tedious aspect of morphing. For feature correspondence between two images, a warp function over the whole image plane must be derived. This process is referred warp generation, and it is an interpolation problem. Warping determines the way in which the pixels in one image should be mapped to the pixels in the other image [5]. Another interesting issue in image morphing is transition control. If transition rates are allowed to vary locally across in between images, more interesting animations are possible [2][8][9].

In this paper i have tried to compare two morphing techniques based on implementation results on various attributes. Here for comparative study defined attributes are such as Computational Time,

DOI : $10.5121 /$ sipij.2011.2420 
Signal \& Image Processing : An International Journal (SIPIJ) Vol.2, No.4, December 2011

Visual Quality of Morphs obtained and Complexity involved in Selection of features, for making an effective comparison.

The rest of the paper is divided as follows: Section 2 explains the principal behind morphing algorithms, Section 3 deals with various warping techniques with section 3.1 dealing with point Warping with triangulation method, 3.2 follow with line warping with Feature based Morphing method. Section 4 presents our implementation results with analysis study and Section 5 a brief conclusion.

\section{MORPHING PRINCIPAL}

The basic principle behind image morphing is explained in this section. Before the development of morphing, image transitions were generally achieved through the use of cross-dissolves, e.g., linear interpolation to fade from one image to another. Figure I depicts this process applied over ten frames. The result is poor, owing to the double exposure effect apparent in misaligned regions. This problem is particularly apparent in the middle frame, where both input images contribute equally to the output. Morphing achieves a fluid transformation by incorporating warping to maintain geometric alignment throughout the cross-dissolve process [1][2]. So Morphing refers to the combination of generalized image warping with a cross-dissolve between image elements. In order to morph between two images we define corresponding control pixels in source image I0 and destination image I1. We then define each intermediate frame I of the metamorphosis by creating a new set of control pixels by interpolating the control pixels from their positions in I0 to the positions in I1.Both images I0 and I1 are then warped toward the position of the control pixels in I. These two warped images are cross-dissolved throughout the metamorphosis [6]. Therefore the different morphing techniques differ mainly in the way in which they perform warping. In the following section we will describe various image warping techniques.

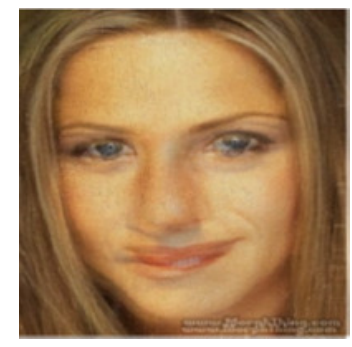

Fig. I. Cross-dissolve Image

\section{WARPING TECHNIQUES}

The process of warping an image can be divided into two steps: First Compute the desired displacements of all pixels in the source image, and second resample the image to create the output image. Resampling can be performed efficiently by processing horizontal and vertical displacements in two separate stages. For the first step it is necessary to construct of a suitable mapping function also called as the deformation step. It is commonly performed either by applying a global analytic mapping function to the image pixel positions or by using a set of control points that specify the displacements of some points in the initial image. In mesh morphing [1][2][12] requires these control points to be the nodes of quadrilateral mesh. In Field morphing [3][13] uses control lines. Scattered data interpolation through triangulation of the data points is a classic approach to scientific visualization [4][9][10][11]. 
Signal \& Image Processing : An International Journal (SIPIJ) Vol.2, No.4, December 2011

\subsection{TRIANGULATION-BASED MORPHING}

This method consists of first dissecting the Definition space into a suitable set of triangles with the given data points as the corners of the triangles. Then, each of the triangles is interpolated independently. Several criteria for an optimal triangulation are known. But Delaunay triangulation maximizes the minimum inner angle of all triangles to avoid thin triangles. It can be computed with a divide-and-conquer algorithm of complexity $\mathrm{O}(\mathrm{n} \log \mathrm{n})$ where $\mathrm{n}$ is the number of data points. If the pixels lie on a regular grid, the correspondence between triangles and pixels is easily known in constant time, and thus each pixel can be mapped to its new location in constant time. With $\mathrm{N}$ the number of pixels in the images, this gives an overall complexity of a continuous, although not smooth, interpolation can be obtained by linear interpolation within each triangle [4][8].

This method has been applied to image warping the visual appearance of the result is quite acceptable if the deformations are small and if enough data points are provided so that changes of the transform coefficients between neighbouring triangles remain small. A smooth deformation can be obtained by using nonlinear patches within the triangles, A problem common to all triangulation-based methods for image warping is that fold over can easily occur. The term fold over describes the occurrence of overlapping deformations, that is, several nonadjacent pixels in the input image are mapped to the same pixel in the output image. With triangulation-based methods, this happens if the orientation of the corner points changes for any of the triangles, that is, the triangle is flipped over by the deformation [4].

Triangulation based Image Morphing Algorithm

Input: Source image, Destination Image

Output: Morphed Image

1. Enter source image, destination image and control points for each image.

2. Divide each image into mesh of triangles using four corners and given control points as vertices.

3. Interpolate between each source and destination triangles pair to generate warped frame.

4. Then warped frame is cross dissolved with given cross dissolve factor.

\subsection{FEATURE BASED IMAGE MORPHING}

This method gives the animator a high level of control over the process. The animator interactively selects corresponding feature lines in the 2 images to be morphed. The algorithm uses lines to relate features in the source image to features in the destination image. It is based upon fields of influence surrounding the feature lines selected. It uses reverse mapping (i.e. it goes through the destination image pixel by pixel, and samples the correct pixel from the source image) for warping the image.

A pair of lines (one defined relative to the source image, the other defined relative to the destination image) defines a mapping from one image to the other. 
Signal \& Image Processing : An International Journal (SIPIJ) Vol.2, No.4, December 2011
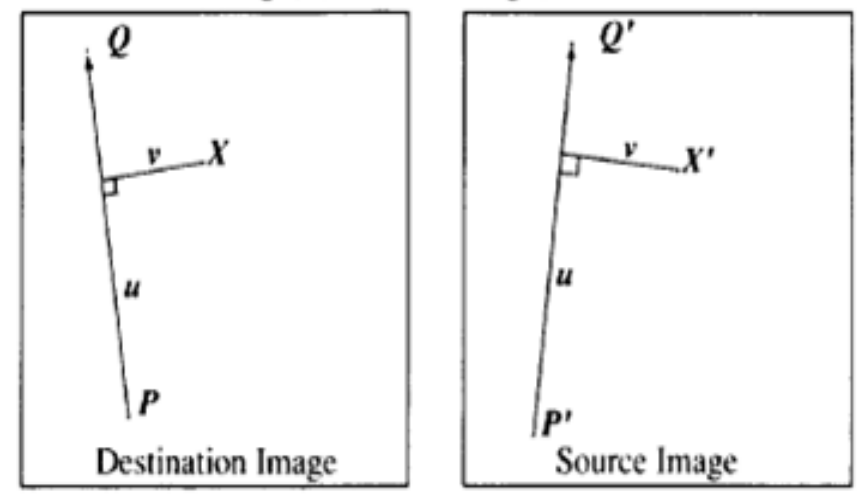

Figure II. Single pair line algorithm[3]

The following parameters are calculated as,

$u=\frac{(X-P) \cdot(Q-P)}{\|Q-P\|^{2}}$

$v=\frac{(X-P) \cdot P \text { erpendizulur }(Q-P)}{\|Q-P\|}$

$X^{\prime}=P^{\prime}+u \cdot\left(Q^{\prime}-P^{\prime}\right)+\frac{\text { v. Perpendicular }\left(Q^{\prime}-P^{\prime}\right)}{\left\|Q^{\prime}-P^{\prime}\right\|}$

Where $\mathrm{X}$ is the pixel co-ordinate in the destination image and $\mathrm{X}^{\prime}$ is the corresponding pixel coordinate in the source image, $\mathrm{PQ}$ is a line segment in the destination image and P' $\mathrm{Q}$ ' is the corresponding line segment in the source image, $\mathrm{u}$ is the position along the line, and $\mathrm{v}$ is the distance from the line. The value $\mathrm{u}$ goes from 0 to 1 as the pixel moves from $\mathrm{P}$ to $\mathrm{Q}$, and is less than 0 or greater than 1 outside that range. The value for $\mathrm{v}$ is the perpendicular distance in pixels from the line. The above algorithm is for the case of a single feature line. In a normal morphing scenario, however there are multiple features in the images to be morphed and consequently multiple feature line pairs are specified.

The displacement of a point in the source image is then, actually a weighted sum of the mappings due to each line pair, with the weights attributed to distance and line length. The weight assigned to each line should be strongest when the pixel is exactly on the line, and weaker the further the pixel is from it. The equation used is as follow.

$$
\text { weight }=\left(\frac{\text { length }^{p}}{(a+\text { dist })}\right)^{b}
$$

Feature Based Image Morphing Algorithm

Input: Source Image and Destination Image

Output: Morphed Image

Step 1: Select lines in source image (I0) and Destination Image (I1).

Step 2: Generate intermediate frame I by generating new set of line segments by interpolating lines from their positions in I0 to positions in I1. 
Signal \& Image Processing : An International Journal (SIPIJ) Vol.2, No.4, December 2011

Step 3: now intermediate frame I pixels in each line segments map to frame I0 pixels by multiple line algorithm.

Step 4: Multiple line algorithm

For each pixel $\mathrm{X}$ in the destination

$\operatorname{DSUM}=(0,0)$

Weight sum $=0$

For each line $\mathrm{P}_{\mathrm{i}} \mathrm{Q}_{\mathrm{i}}$

Calculate $\mathrm{u}$, v based on $\mathrm{P}_{\mathrm{i}} \mathrm{Q}_{\mathrm{i}}$

Calculate $\mathrm{X}^{\prime} \mathrm{i}$ based on $\mathrm{u}, \mathrm{v}$ and $\mathrm{P}_{\mathrm{i}}^{\prime} \mathrm{Q}_{\mathrm{i}}{ }^{\prime}$

Calculate displacement $\mathrm{D}_{\mathrm{i}}=\mathrm{Xi}-\mathrm{Xi}$

dist $=$ shortest distance from $X$ to $P_{i} Q_{i}$

$$
\text { weight }=\left(\frac{\operatorname{length}^{p}}{(a+d i s t)}\right)^{k}
$$

$$
\text { DSUM += Di } * \text { weight }
$$

Weight sum $+=$ weight

$\mathrm{X}^{\prime}=\mathrm{X}+\mathrm{DSUM}$ / weight sum

Destination Image $(X)=$ Source Image $\left(X^{\prime}\right)$

Step 5: then intermediate frame I pixels in each line segments map to frame I1 pixels by multiple line algorithm.

Step 6: now warped image I0 and warped image I1 is cross dissolved with given dissolution factor. Dissolution factor will be in [0 1].
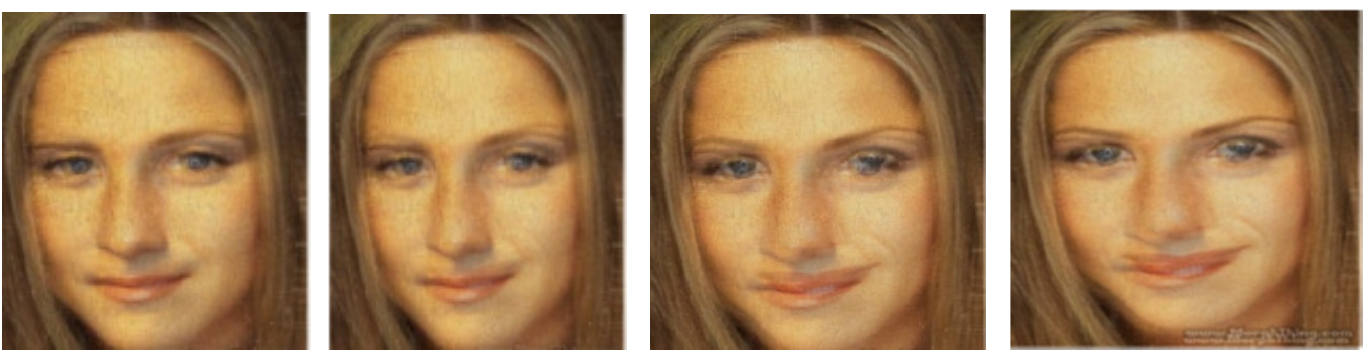

Source Image
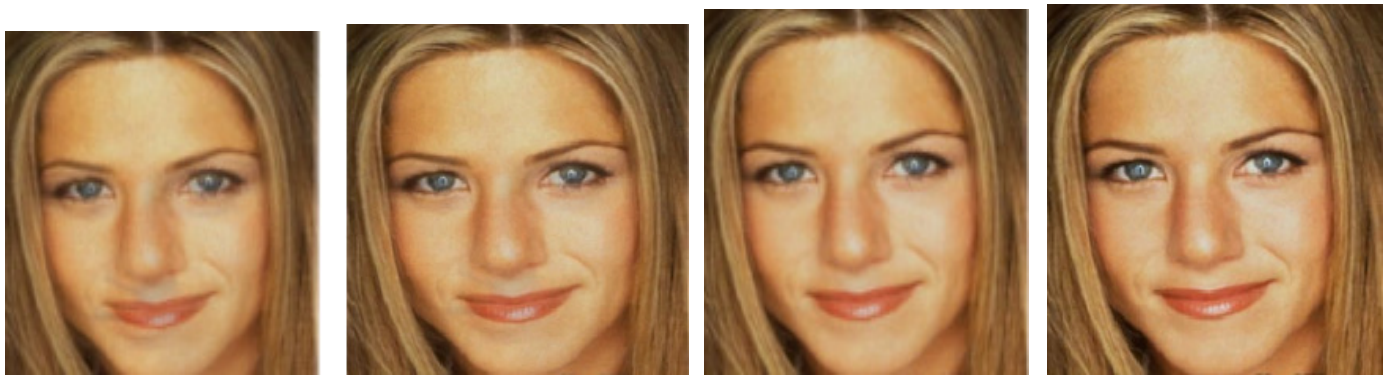

Destination Image

(a) Cross - Dissolve 
Signal \& Image Processing : An International Journal (SIPIJ) Vol.2, No.4, December 2011
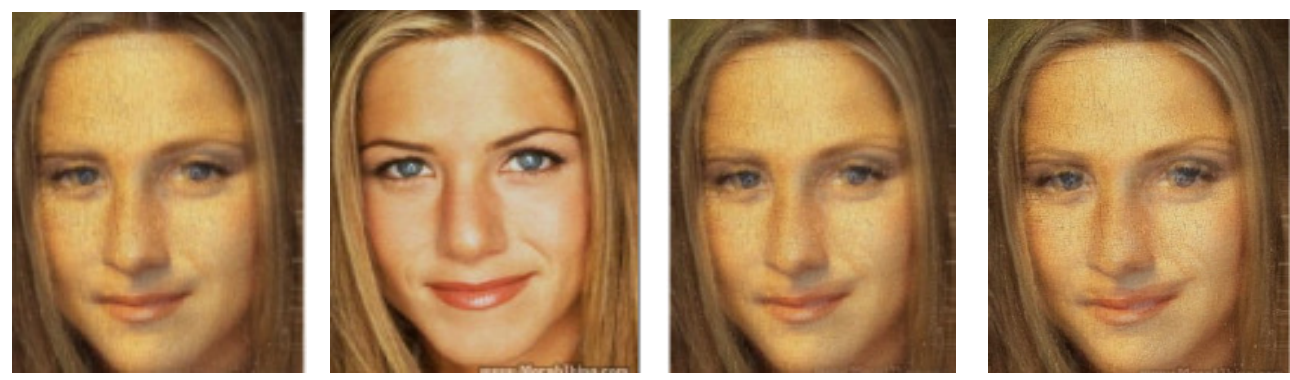

Source Image
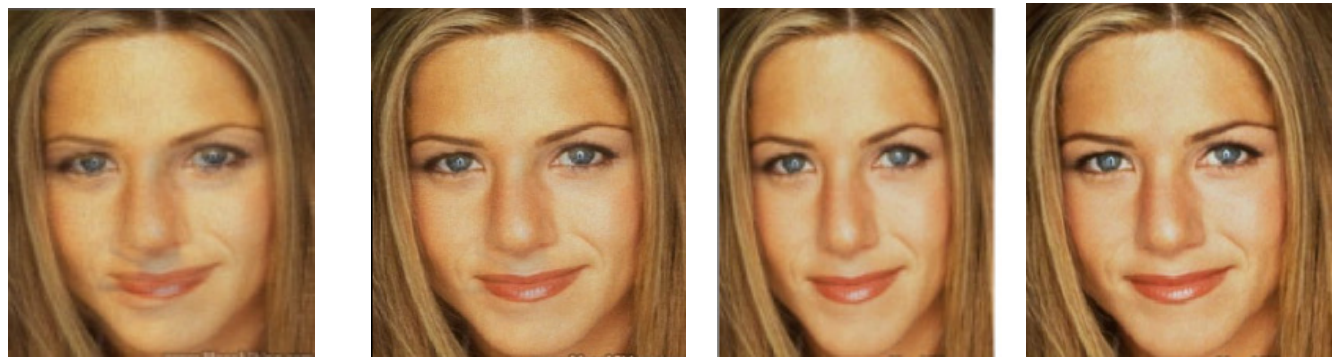

Destination Image

(b)Triangulation based Morphing
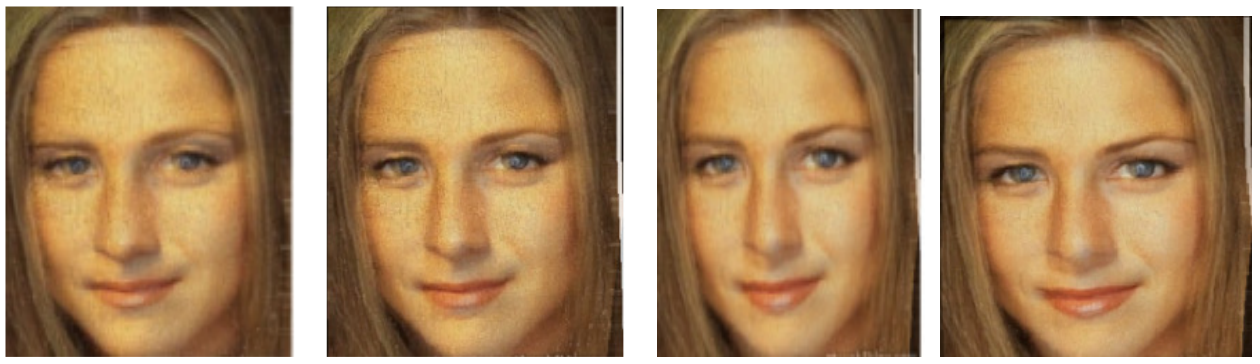

Source Image
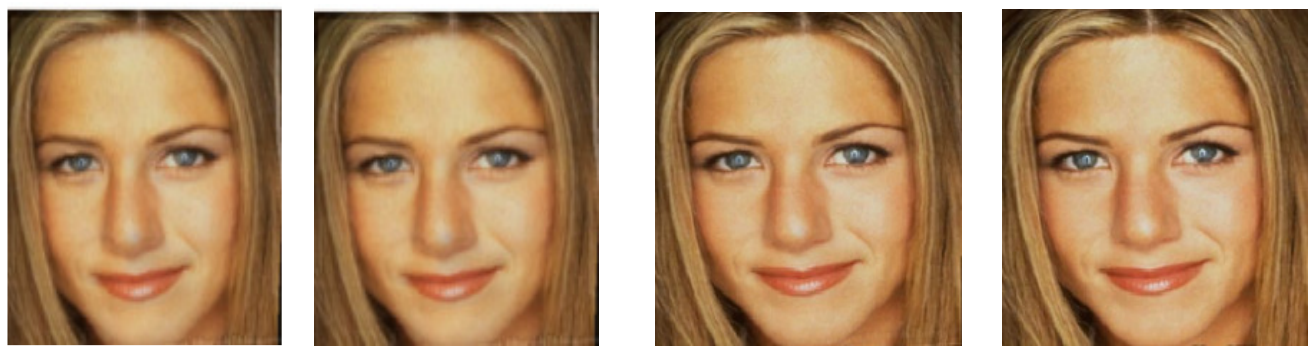

Destination Image

(c ) Feature based Morphing

Figure III: Results of Morphing using (a) Simple Cross Dissolve (b) Triangulation based Morphing (c ) Feature based Morphing 
Signal \& Image Processing : An International Journal (SIPIJ) Vol.2, No.4, December 2011

\section{RESULTS}

Figure III illustrates the results of the morphing procedure using just cross dissolve and also with triangulation based Morphing algorithms and Feature based Image Morphing algorithm. It is evident from looking at Figure III. that the morphs obtained by just cross dissolving the images without warping them is very bad. This is because features of the source and destination will not be aligned. Double Exposure is evident in the misaligned regions. From the results obtained we can also see that the quality of morph obtained using Triangulation based warping is more natural compare to simple cross dissolve. But computational complexity is high in this method. Feature based Morphing have natural results compare to Triangulation Morphing. Also Feature morphing gives good results for different orientation images. While triangulation morphing gives good results for same orientation images.

Triangulation-based methods have several disadvantages: They have non-contiguous derivatives, and they are not defined outside the convex hull of the control points. In addition, for aesthetically pleasing results, they need a large number of triangles. Also appropriate control points should be selected by animator. In feature morphing with less feature points also it gives good morphing results. So ease of animator point of view feature morphing is good compare to triangulation morphing. Feature morphing has disadvantage of "ghost" lines. It can be corrected by adding some lines or deleting some lines. Feature morphing gives high level of control to programmer on output results. Also computational complexity is high in triangulation method compare to feature morphing.

For triangulation Based Image Morphing 6 feature points with triangles are shown in Figure IV. Algorithm make sub triangles of whole image considering feature points and image corner points. Also Figure V. shows 4 feature lines selected by animator for feature based image morphing algorithm.

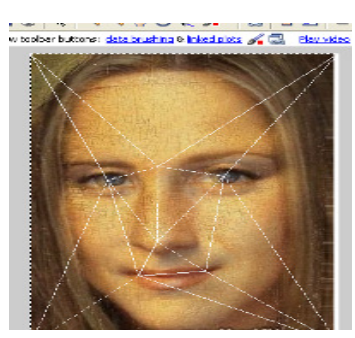

Source Image

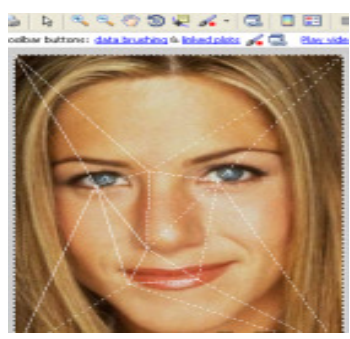

Destination Image

Figure IV: Triangulation Morphing with 6 Feature Points

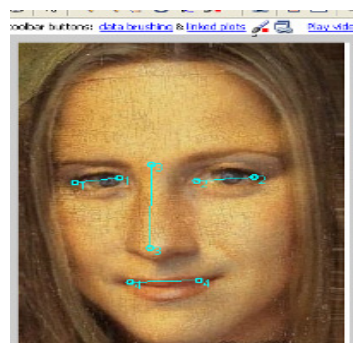

Source Image

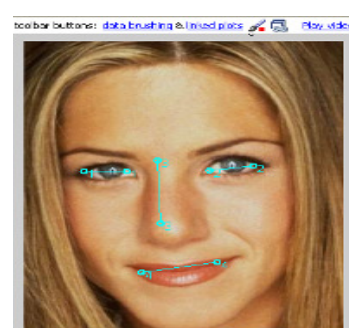

Destination Image

Figure V: Feature Morphing with 4 Feature Lines 
Signal \& Image Processing : An International Journal (SIPIJ) Vol.2, No.4, December 2011

In Triangulation based method for better visual quality of morph require more number of triangulations so require more control points. Hence, we feel that for a given quality of morph results the Feature based Morphing algorithm needs least effort on part of the animator.

After implementing this method in MATLAB Finally we wish to put in a word on the individual advantage and disadvantages of this algorithms. The triangulation based warping algorithm are automatic and create good morphing effect for same orientation images. But require that control points selected such that it do not create fold over problem, because fold over problem degrade quality of morph Images. Feature morphing requires less control lines by animator and gives natural morphing for different orientation images.

\section{CONCLUSION}

The focus of this paper has been to discuss image Morphing terms and survey various morphing algorithms and provide the comparative study of triangulation based morphing algorithm and feature based image morphing algorithm implementation details with advantage and disadvantage. It is found that triangulation based morphing algorithms are complex to implement and it requires a significant amount of time compare to feature based morphing algorithm. Also visual morphed results are natural for different orientation images in feature morphing and also it require to provide less control lines appropriately by animator. So to reduce work of animator for morphing for face images, key features of face such as noise tip, eyes points and lip points can be extracted by automatic processing which make this morphing as automatic image morphing. There are a variety of Image Morphing algorithms such as Thin Plate Spline Image Morphing, Image Morphing with snakes and free formed deformations, Image morphing using deformable surfaces and many others besides. Due to paucity of resources and time, unable to provide a comprehensive comparison of these algorithms.

\section{REFERENCES}

[1] George Wolberg "Recent Advances in Image Morphing" Department of Computer Science City College of New York.

[2] Wolberg, G., "Image Morphing: A Survey”, Visual Computer (2278), vol. 10, pp. 3150-225.

[3] Beier T., And Neely, S.,"Feature-based image metamorphosis", Proc. SIGRAPH 92 Computer Graphics (2272), pp. 35-102.

[4] D. Ruprecht and H. Muller, "Image warping with scattered data interpolation",IEEE Computer Graphics and Applications, 15(2):23-103, March 2275.

[5] Pramod Potluri ,Krishna Sagiraju and Venkatachalam Tubati "Image Morphing: Feature based, View and Mesh" Clemson University, Clemson.

[6] Prashant K. Oswal and Prashanth Y. Govindaraju "Image Morphing: A Comparative Study", Department of Electrical and Computer Engineering, Clemson University, Clemson.

[7] Wolberg, G., "Digital Image Warping", IEEE Computer Society Press, Los Alamitos, CA, 2270.

[8] Ayellet Tal, Gershon Elber,“ Image Morphing with feature preserving Texture” EUROGRAPHICS '99 vloume 18(2279) Number 3

[9] Lee S.Y., Chwa K.Y,Shin S.Y., Wolberg G. , "Image Metamorphosis Using Snakes and Free-Form Deformations", Computer Graphics (2275) 
Signal \& Image Processing : An International Journal (SIPIJ) Vol.2, No.4, December 2011

[10] D. Ruprecht and H. Muller, "Image warping with scattered data interpolation", IEEE Computer Graphics and Applications,15(2):23-103, March 2275.

[11] Bookstein F. L, "Principal Warps: Thin-plates splines and decomposition of deformations", IEEE Trans. Pattern Analysis and Machine Intelligence (2289), vol 1(15), pp. 5157-585.

[12] Seitz, S.M., and Dyer C. R, "View Morphing”, Proc. SIGRAPH 915. Computer Graphics (22715), pp. 21-28.

[13] Srinath Setty, Swati Rallapalli, fsrinath, "Implementation of Morphing algorithm with Feature-based Image metamorphosis", The University of Texas, Austin.

\section{Author}

Assistant Professor Ms. Bhumika G. Bhatt has Received her BE (Information Technology) Degree from G. H. Patel College of Engg \& Technology, Vidyanagar, Gujarat, India in year 2000 and ME ( Computer Engg ) from BVM Engg. College, Gujarat Technological University in year 2011. She has more than $8+$ years of Teaching experience in education field. Currently, she is working as an Assistant Professor in the Department of Computer Engineering at Vidyabharti Trust Institute of Technology \& Research Center, Bardoli, Gujarat, India. She has published papers in National/International Conferences and International Journals. Her main research interests involve Digital Image Processing, Neural Network and Fuzzy Logic, Operating

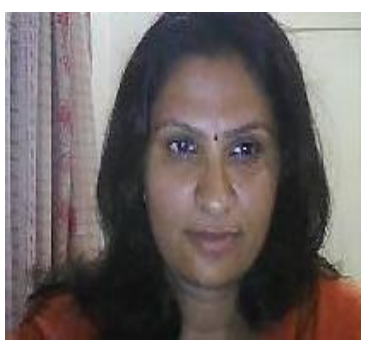
Systems and Computer Algorithms. 